\title{
Six Percent Incidence of Graft-Tunnel Mismatch in Anatomic Anterior Cruciate Ligament Reconstruction Using Bone-Patella Tendon-Bone Autograft and Anteromedial Portal Drilling
}

\author{
Tim Dwyer, M.B.B.S., Ph.D., Graeme Hoit, M.D., Michael Sellan, M.D., \\ Rohit Gopinath, B.Sc., Daniel Whelan, M.D., M.Sc., John Theodoropoulos, M.D., M.Sc., and \\ Jaskarndip Chahal, M.D., M.Sc.
}

\begin{abstract}
Purpose: The purpose of this study was to determine the incidence of graft-tunnel mismatch (GTM) when performing anatomic anterior cruciate ligament reconstruction (ACLR) using bone-patella tendon-bone (BPTB) grafts and anteromedial portal drilling. Methods: Beginning in November 2018, 100 consecutive patients who underwent ACLR by two sports fellowship-trained, orthopedic surgeons using BPTB autograft and anteromedial portal drilling were prospectively identified. The BPTB graft dimensions and the femoral tunnel distance, tibial tunnel distance, intra-articular distance, and total distance were measured. Surgeons determined the depth and angle of tunnels based on the patella tendon graft length dimensions in each case. After passage of the graft, the distance from the distal graft tip to the tibial cortex aperture was measured. GTM was defined as the need for additional measures to obtain satisfactory tibial graft fixation $(<15-20$ $\mathrm{mm}$ of bone fixation). Results: The incidence of mismatch was $6 / 100(6 \%)$. Five cases involved the graft being too long, with the tibial bone plug protruding excessively from the tibial tunnel- $4 / 5$ had a patella tendon length $\geq 50 \mathrm{~mm}$. Three cases were managed with femoral tunnel recession, and two were treated with a free bone plug technique. One patient with a patella tendon length of $35 \mathrm{~mm}$ had a graft that was too short, with the tibial bone plug recessed in the tibial tunnel. Of patients whose tibial tunnel distance was within $5 \mathrm{~mm}$ of the patella tendon length, only $1 / 46(2 \%)$ patients had mismatch, whereas $5 / 54(9 \%)$ of patients who had $>5 \mathrm{~mm}$ difference had mismatch. Conclusions: The incidence of graft-tunnel mismatch after anatomic ACLR using BTPB and anteromedial portal drilling in this study is 6\%. To limit the occurrence of GTM where the graft is too long, surgeons should drill tibial tunnel distances within $5 \mathrm{~mm}$ of the patella tendon length. Clinical Relevance: The results of this study provide surgeons with a technique of limiting graft tunnel mismatch when performing ACLR using BPTB and anteromedial portal drilling.
\end{abstract}

\section{Introduction}

A $\mathrm{n}$ important technical consideration for surgeons performing anterior cruciate ligament reconstruction (ACLR) using bone-patella-tendon-bone

University of Toronto Orthopaedic Sports Medicine, Toronto, Ontario, Canada (T.D., G.H., M.S., R.G., D.W., J.T., J.C); Women's College Hospital, Toronto, Ontario, Canada (T.D., D.W., J.T., J.C.); and Mt. Sinai Hospital, Toronto, Ontario, Canada (T.D., J.T.).

Full ICMJE author disclosure forms are available for this article online, as supplementary material.

Received April 11, 2021; accepted November 4, 2021.

Address correspondence to Tim Dwyer, M.B.B.S., Ph.D., Women's College Hospital, 76 Grenville St., Toronto, Canada,M5S 1B2.E-mail: tim.dwyer@ wchospital.ca

(C) 2021 THE AUTHORS. Published by Elsevier Inc. on behalf of the Arthroscopy Association of North America. This is an open access article under the CC BY-NC-ND license (http://creativecommons.org/licenses/by-nc-nd/4.0/). 2666-061X/21543

https://doi.org/10.1016/j.asmr.2021.11.006
(BPTB) ACL reconstruction is graft-tunnel mismatch (GTM). GTM occurs when there is a significant difference between the total length of the BPTB graft, and the total distance of the femoral tunnel distance (FTD), intraarticular distance (IAD), and the tibial tunnel distance (TTD). ${ }^{1}$ The combination of a long graft and short tunnels can result in a tibial bone plug that protrudes out of the tunnel, compromising adequate bony fixation on the tibial side. ${ }^{2,3}$ Short grafts with relatively long tunnels can also cause bony fixation issues on the tibial side, including screw divergence and graft advancement or laceration. ${ }^{4}$ Although it is accepted that mismatch is more common when using allograft in comparison to autograft, ${ }^{2,5}$ GTM using autograft has been described when using transtibial drilling techniques. ${ }^{2,6}$

The rate of autograft BPTB mismatch using endoscopic techniques and transtibial drilling has been reported to be between $10 \%{ }^{2}$ and $26 \%{ }^{6}$. However, over the past 

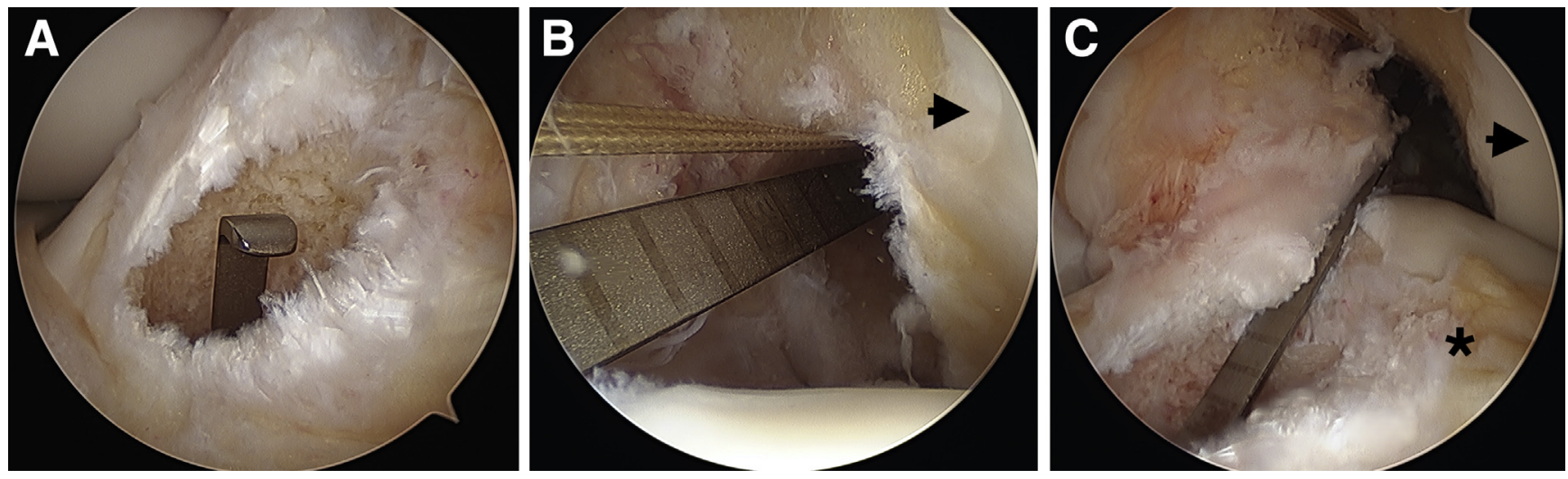

Fig 1. (A) Arthroscopic measurement of the tibial tunnel using EndoButton depth gauge. (B) Arthroscopic measurement of the femoral tunnel, performed through an accessory anteromedial portal with the knee hyperflexed. Ar denotes lateral femoral condyle. (C) Intra-articular distance between the tibial tunnel and the femoral tunnel, with the knee at $30^{\circ}$ of flexion. Ar denotes lateral femoral condyle. Asterisk denotes lateral tibial plateau (Reproduced with permission). Images are of a left knee, with the camera in the anterolateral portal.

two decades, there has been increased use of anteromedial portal methods to drill femoral tunnels in ACLR. This shift in technique is in accordance with evidence that anteromedial portal drilling can change the position, length, and obliquity of the femoral tunnel in comparison to transtibial drilling, ${ }^{7-9}$ position the tunnel at the center of the native ACL attachment site, ${ }^{10,11}$ recreate the anisometry of the native ACL compared to transtibial techniques ${ }^{12,13}$, and thus, potentially influence mismatch ${ }^{14,15}$, although evidence of clinically superior outcomes is lacking. ${ }^{16}$ However, the true incidence of mismatch using anteromedial drilling techniques is unknown.

The ability to recognize and manage GTM is clearly important for surgeons who perform anatomic ACLR using BPTB grafts. A previous study using cadavers demonstrated that measurements of FTD, TTD, and the IAD between these two tunnels were reliable. ${ }^{17}$ The purpose of this study was to determine the incidence of GTM when performing anatomic ACLR using BPTB grafts and anteromedial portal drilling. We hypothesized that the incidence of GTM would be low when performed by surgeons experienced in ACLR using BTPB and anteromedial portal drilling.

\section{Methods}

Beginning in November 2018, 100 consecutive patients who underwent ACLR by two sports fellowshiptrained orthopedic surgeons (T.D., J.C.) using BPTB autograft and anteromedial portal drilling were prospectively identified. This study was formally reviewed by Women's College Hospital and deemed not to require Research Ethics Board approval (IRB; REB \# 2019-0068-E). Inclusion criteria were any patient undergoing ACLR using BPTB autograft and performed by way of anteromedial portal drilling between the ages of 14 and 60, who were skeletally mature. Exclusion criteria were skeletally immature patients and patients undergoing concomitant ligament reconstruction.

For each case, the dimensions of the bone graft were measured after harvest (patella bone plug length, patella tendon length, tibial bone plug length and diameter, and total graft length). Surgeons also performed arthroscopic measurements of FTD, TTD, and IAD at both $30^{\circ}$ and $90^{\circ}$. They also measured total distance after the femoral and tibial tunnels had been drilled, using an EndoButton depth gauge placed in the center of the tunnels, as per a previously described technique $^{17}$ (Fig 1).

Both surgeons used similar graft techniques, taking a $20 \mathrm{~mm} \times 9 \mathrm{~mm}$ patella bone plug, and a $25-30 \mathrm{~mm} \times 9$ $\mathrm{mm}$ tibial bone plug. The tibial bone plug was made 5$10 \mathrm{~mm}$ longer in order to avoid GTM, where the graft is too short. Both surgeons used similar drilling techniques, drilling the femoral tunnel to $25 \mathrm{~mm}$ using the anteromedial portal with the knee hyperflexed $\left(\sim 120^{\circ}\right)$, and performing ACLR with metal screw fixation on both sides. The femoral tunnel was placed within the footprint of the previous ACL, using a 7-mm offset guide to ensure a 2 -mm back wall.

In an effort to avoid mismatch, both surgeons adjusted their techniques depending on the length of the patella tendon, each drilling the tibial tunnel first. Both surgeons used Smith \& Nephew Acufex Director Drill Guide (Andover, MA) for tibial tunnel drilling. Surgeon 1 (J.C.) varied the tibial tunnel length as follows: 1) if the length of the patellar tendon was less than $50 \mathrm{~mm}$, the start point for the tibial tunnel was $\sim 4$ $\mathrm{cm}$ from the joint line with a $60^{\circ}$ tibial aimer; and 2) when the tendon length was $50 \mathrm{~mm}$ or greater, the start point on the tibia was $5-6 \mathrm{~cm}$ from the joint line using a $65^{\circ}$ tibial aimer. Surgeon 1 would drill the tibial tunnel within a single anteromedial incision that was also used for graft harvest and placement of the anteromedial 


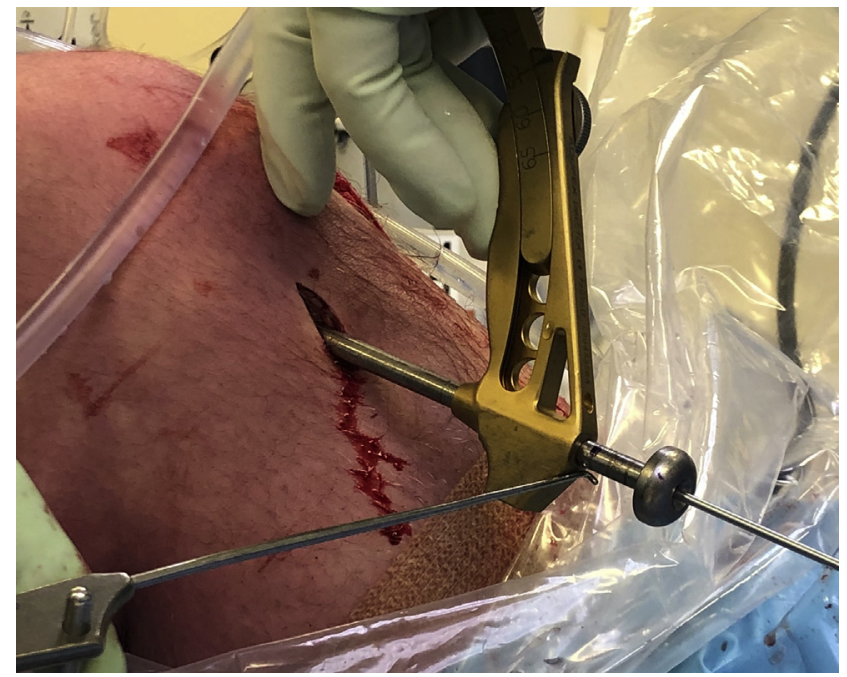

Fig 2. Surgeon 2 using the Smith $\&$ Nephew Acufex Director Drill Guide (Andover, MA) to estimate the tibial tunnel distance, aiming for a distance within $5 \mathrm{~mm}$ of the patella tendon distance. Image is of a left knee, with the camera in the anterolateral portal.

portal. Surgeon 1 would also overdrill the femoral tunnel a priori between 25 and $30 \mathrm{~mm}$ for patella tendon lengths greater than $50 \mathrm{~mm}$.

Surgeon 2 (TD) also varied the tibial tunnel length based on the length of the patella tendon, with the tibial aimer set at $60^{\circ}$ for a patella tendon graft $<50 \mathrm{~mm}$, and set at $65^{\circ}$ for a patella tendon graft $>50 \mathrm{~mm}$. However, Surgeon 2 used the measurements on the angled bullet of the tibial angled guide to estimate the tunnel length prior to drilling, aiming for a TTD within $5-10 \mathrm{~mm}$ of the patella tendon length by altering the tibial starting point as required (Fig 2). Surgeon 2 also made a separate incision for the tibial tunnel, to limit the extent of the bone graft incision.

After passage of the graft, we measured the length of bone protruding from or recessed into the tibial tunnel from the tibial cortex to the distal tip of the graft at $0^{\circ}$, $30^{\circ}$, and $90^{\circ}$ (Fig 3). The amount of bone plug in the tibial tunnel was calculated by deducting the length of bone protruding from the measured length of tibial bone plug. GTM was defined as the need for additional surgical measures to obtain satisfactory graft fixation on the tibial side, when $<15$ to $20 \mathrm{~mm}$ of bone fixation would be obtained on the tibial side. Both surgeons performed tibial fixation with the knee flexion angle at $15^{\circ}$.

\section{Statistical Analysis}

Means, standard deviations, and ranges were calculated for continuous variables, as appropriate. Categorical variables were presented as counts and proportions. The incidence of mismatch was used as the primary outcome. A sample size calculation was not performed, as the incidence of mismatch using anatomical reconstruction was unknown. All statistical analyses were conducted using SAS v9.4 (SAS Institute, Cary, NC).

\section{Results}

Between November 2018 and February 2020, 100 consecutive patients were included in this prospective study, with a mean age of 26.4 years (range: 14-51); 44 patients were female. The graft characteristics are presented in Table 1 , and the tunnel characteristics are presented in Table 2.

With regard to mismatch, the mean graft length protruding from the tibial tunnel at $90^{\circ}$ was $9.2 \mathrm{~mm}$ ( \pm 4.8 ; range: $0-28 \mathrm{~mm}$ ), the mean graft protruding at $30^{\circ}$ was $8.1 \mathrm{~mm}( \pm 5.0$, range: $-2-28 \mathrm{~mm})$, and the mean graft protruding at $0^{\circ}$ was $6.5 \mathrm{~mm}( \pm 5.2$, range: $-4-28 \mathrm{~mm})$. The mean excursion of the graft construct from $90^{\circ}$ to full extension was $2.7 \mathrm{~mm}( \pm 2.4$, range: $0-9 \mathrm{~mm}$ ). The mean excursion of the graft from 0 to $30^{\circ}$ was $1.5 \mathrm{~mm}( \pm 1.5$, range: $0-6 \mathrm{~mm})$ and from $30-90^{\circ}$ was $1.2 \mathrm{~mm}( \pm 1.4$, range: $0-5 \mathrm{~mm})$.

Out of the 100 cases, there were 6 cases of mismatch $(6 \%)$, where the surgeons had to change technique in order to get adequate fixation (Table 3 ). Five of the six $(83 \%)$ cases of GTM involved the graft being too long

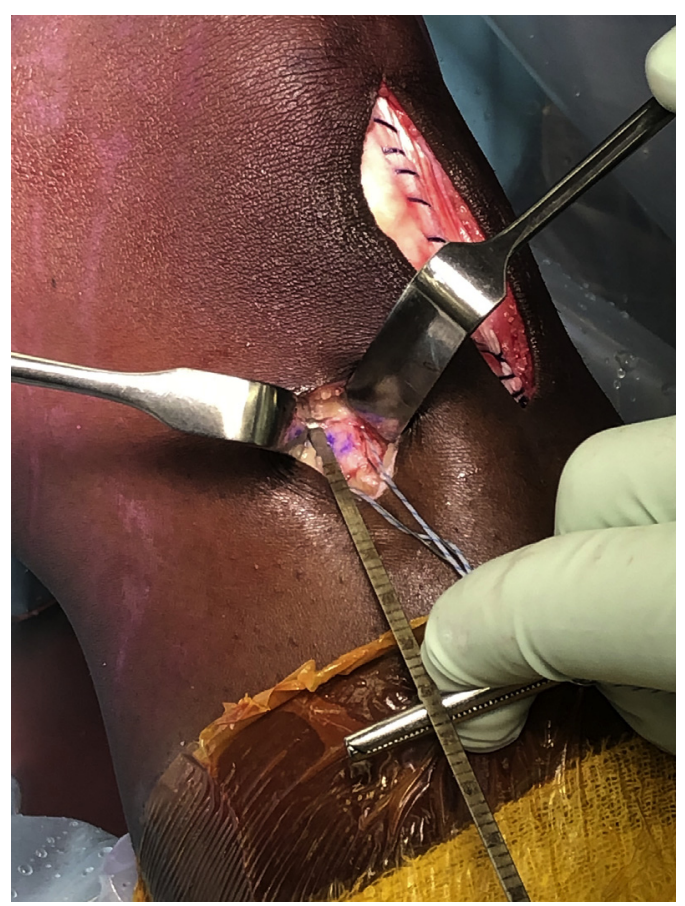

Fig 3. Surgeon 2 using the EndoButton depth gauge to measure the amount of bone protruding prior to tibial fixation in a left knee at $30^{\circ}$ of flexion. In this case, $12 \mathrm{~mm}$ of a $32-\mathrm{mm}$ tibial bone block was protruding, leaving $20 \mathrm{~mm}$ of bone in the tibial tunnel. Accordingly, this was not a case of mismatch, as adequate tibial fixation could be obtained. 
Table 1. Graft Characteristics

\begin{tabular}{lcc}
\hline \multicolumn{1}{c}{ Measurement } & Mean $(\mathrm{SD}), \mathrm{mm}$ & Range \\
\hline Patella bone plug length & $20.5( \pm 1.6)$ & $15-25$ \\
Tibial bone plug length & $28.6( \pm 2.6)$ & $20-36$ \\
Patella tendon length & $44.6( \pm 6.1)$ & $29-61$ \\
Total graft length & $93.7( \pm 6.4)$ & $79-113$ \\
\hline
\end{tabular}

and protruding from the tibial tunnel-only one case of the graft being too short was encountered.

Among the 5 cases with GTM with the graft too long, the mean length of the patellar tendon was $51.4 \mathrm{~mm}$ (range: 46-55). Overall, 23 patients had a patella tendon length $\geq 50 \mathrm{~mm}$, with a mean length of 52.8 mm (range: 50-61), a mean TTD of 42.6 (range: $34-52$ ), and a mean tibial bone block of 28.4 (range: 25-31). Among those patients whose patella tendon lengths were $\geq 50 \mathrm{~mm}, 17 \%(4 / 23)$ had a mismatch, with a mean patella tendon length of 52.8 (range: 50-55), a mean TTD of 39 (range: 36-46) and a mean tibial bone block of 29.3 (range: 28-30). Among those patients whose patella tendon lengths were $\geq 50 \mathrm{~mm}, 19 / 23$ $(83 \%)$ did not have mismatch, with a mean patella tendon length of 52.8 (range: 50-61), a mean TTD of 43.2 (range: $34-52$ ), and a mean tibial bone block of 28.4 (range: $25-31$ ).

Of patients whose tibial tunnel distance was within 5 $\mathrm{mm}$ of the patella tendon length, only $1 / 46(2 \%)$ patients had mismatch, whereas 5/54 (9\%) of patients who had $>5 \mathrm{~mm}$ difference had mismatch (mean 13 mm, range: 9-19). In the single case where the graft was too short, the TTD was greater than the patella tendon length. There were no cases of femoral tunnel blowout of the back wall or short femoral tunnels.

\section{Discussion}

In this prospective cohort study, we found the incidence GTM to be $6 \%$ of ACLR patients. Our findings demonstrate that GTM is a concern when performing anatomic ACLR using BPTB and anteromedial portal drilling, even when surgeons are acutely aware of the potential risk. Importantly, we have also identified a low rate of GTM when the tibial tunnel length was within 5 $\mathrm{mm}$ of the patella tendon length-emphasizing that surgeons must plan tunnels based upon patella tendon lengths in order to prevent GTM. Surgeons must also have a firm understanding of tunnel modification, graft modification, and alternative fixation techniques to rectify mismatch when it does occur.

A minimum 10-mm bone plug has been recommended for acceptable graft fixation, ${ }^{18}$ but a protruding graft with less than $20 \mathrm{~mm}$ of tibial bone plug within the tibial tunnel can compromise screw fixation. ${ }^{19}$ Options in this setting include femoral tunnel recession, ${ }^{20}$ graft rotation of between $540^{\circ}$ and $630^{\circ},{ }^{2,21}$ the use of free bone blocks, ${ }^{2,19,22}$ creation of a bony trough and staple fixation of the tibial plug, ${ }^{23,24}$ single bone plug techniques, ${ }^{25}$ folding the bone plug over the patella tendon, ${ }^{26}$ as well as using alternative forms of fixation on the tibia such a screw post fixation. ${ }^{22,27}$

In our study, it was more common to have grafts that were protruding, or too long, as the cause of GTM. In four patients, the protrusion was felt to be relatively small-in these cases, the graft was removed, and the femoral tunnel drilled a further $5-10 \mathrm{~mm}$ in order to obtain 15-20 mm of tibial bone fixation. ${ }^{20,28}$ Although there is some concern that deepening the femoral tunnel may compromise the soft tissue portion of the graft, either by a windscreen washer/abrasion effect, ${ }^{29}$ or by potential damage from the interference screw, ${ }^{30}$ there is no clinical evidence of increased graft failure when using this technique. ${ }^{20}$ It is important to note that in the setting of femoral tunnel recession, a standard $20-\mathrm{mm}$ screw must either be buried in order to interface with the bone block, or a longer screw must be used.

However, femoral tunnel recession has its limitations, especially in the setting of GTM more than $10 \mathrm{~mm}$. In our study, there were two cases in which nearly the entire tibial bone block was protruding, and a free bone block technique was used ${ }^{22}$ (Fig 4). In this technique, the tibial bone plug is excised, and a Krackow type suture performed on the patella tendon-the free bone block is then impacted over the top of the patella tendon in the tibial tunnel, followed by screw fixation. ${ }^{22}$ It is important to place sutures through the free bone block, to prevent proximal migration of the bone during screw insertion.

Likewise, having a graft that is too short can lead to blind placement of screws, with potential inadequate distal fixation. In the one case identified in our series, the graft was $5 \mathrm{~mm}$ too short-with the knee at $30^{\circ}$, the tibial screw was inserted, and satisfactory graft fixation was obtained. Fortunately, full extension was obtained postfixation. Both surgeons in our study took an extra $10 \mathrm{~mm}$ on the tibial bone plug in order to prevent this serious complication. Rescue options for grafts that are too short typically involve tying sutures over a unicortical screw and washer, ${ }^{4}$ which is thought to be an inferior method of fixation. ${ }^{19}$

Most cases of GTM arise from the high degree of variability of patella tendon lengths, with the incidence

Table 2. Tunnel Characteristics

\begin{tabular}{lcc}
\hline \multicolumn{1}{c}{ Measurement } & Mean (SD) & Range \\
\hline Femoral tunnel distance, in mm & $25.9(+2.5)$ & $20-34$ \\
Intra- articular distance at $30^{\circ}$, in mm & $23.4( \pm 2.5)$ & $18-31$ \\
Intra-articular distance at $90^{\circ}$, in mm & $21.9( \pm 2.7)$ & $16-32$ \\
Tibial tunnel distance, in mm & $38.8( \pm 4.5)$ & $28-52$ \\
Tibial drill guide angle, in degrees & $60^{\circ}( \pm 1.9)$ & $50-65$ \\
Total tunnel distance at $30^{\circ}$, in mm & $88.1( \pm 6.3)$ & $73-105$ \\
Total tunnel distance at $90^{\circ}$, in mm & $86.6( \pm 6.1)$ & $72-102$ \\
\hline
\end{tabular}


Table 3. Details of Cases with Graft-Tunnel Mismatch

\begin{tabular}{|c|c|c|c|c|c|c|c|c|c|c|}
\hline & Problem & $\begin{array}{l}\text { FTD, } \\
\text { mm }\end{array}$ & $\begin{array}{l}\text { IAD } \\
\text { at } 30^{\circ}, \\
\text { mm }\end{array}$ & $\begin{array}{l}\text { TTD, } \\
\mathrm{mm}\end{array}$ & $\begin{array}{l}\text { Total } \\
\text { Distance } \\
\text { at } 30^{\circ}, \\
\mathrm{mm}\end{array}$ & $\begin{array}{l}\text { Total } \\
\text { Graft } \\
\text { Length, } \\
\text { mm }\end{array}$ & $\begin{array}{l}\text { Patella } \\
\text { Bone } \\
\text { Plug, } \\
\text { mm }\end{array}$ & $\begin{array}{l}\text { Tibial } \\
\text { Bone } \\
\text { Plug, } \\
\text { mm }\end{array}$ & $\begin{array}{l}\text { Patella } \\
\text { Tendon } \\
\text { Length, } \\
\text { mm }\end{array}$ & Solution \\
\hline Case 3 & $\begin{array}{l}\text { At } 30^{\circ} \text {, the tibial bone plug } \\
\text { was protruding by } 15 \\
\mathrm{~mm} \text {, and by } 15 \mathrm{~mm} \text { at } \\
0^{\circ} \text {. }\end{array}$ & 20 & 24 & 46 & 90 & 105 & 21 & 29 & 55 & $\begin{array}{l}\text { Femoral tunnel recession } \\
\text { by } 5 \mathrm{~mm}\end{array}$ \\
\hline Case 5 & $\begin{array}{l}\text { At } 30^{\circ} \text {, the tibial bone plug } \\
\text { was protruding by } 17 \\
\mathrm{~mm} \text {, and by } 16 \mathrm{~mm} \text { at } \\
0^{\circ} \text {. }\end{array}$ & 25 & 22 & 38 & 85 & 102 & 22 & 30 & 50 & $\begin{array}{l}\text { Femoral tunnel recession } \\
10 \mathrm{~mm} \text {, insertion of a } \\
\text { longer 30mm screw }\end{array}$ \\
\hline Case 6 & $\begin{array}{l}\text { At } 30^{\circ} \text {, the tibial bone plug } \\
\text { was protruding by } 21 \\
\mathrm{~mm} \text {, and by } 20 \mathrm{~mm} \text { at } \\
0^{\circ} \text {. }\end{array}$ & 25 & 19 & 36 & 80 & 101 & 22 & 28 & 51 & $\begin{array}{l}\text { Free tibial bone block } \\
\text { technique (Fig 3) }\end{array}$ \\
\hline
\end{tabular}

FTD, femoral tunnel distance; IAD, intra-articular distance; TTD, tibial tunnel distance.

of graft mismatch shown to be higher when the tendinous portion of a graft is $\geq 50 \mathrm{~mm}^{6}$ The literature describes a mean tendon length of between 40 and 50 $\mathrm{mm} ;{ }^{6,31}$ however, Denti et al. found that a $9 / 50(18 \%)$ of tendon lengths were $<40 \mathrm{~mm},{ }^{31}$ and Shaffer et al. identified that $14 / 34(41 \%)$ that were $\geq 50 \mathrm{~mm}^{6}$. Certainly, a wide variety was seen in our study, with a mean tendon length of $45 \mathrm{~mm}$, but with a range between 29 and $61 \mathrm{~mm}$. In our study, 17/100 (17\%) were $<40 \mathrm{~mm}$, and $22 / 100(22 \%)$ were $\geq 50 \mathrm{~mm}$. Importantly, 4/6 cases of GTM were encountered with patella tendon lengths of $\geq 50 \mathrm{~mm}$, and the one case where the graft was too short was associated with a patella tendon length of $35 \mathrm{~mm}$. Furthermore, in the five cases of GTM in which the graft was too long, all of these cases had a $>5$-mm difference between the tibial tunnel distance and the patella tendon length-in the two cases requiring a free bone block technique, there was a discrepancy of $15 \mathrm{~mm}$ and $19 \mathrm{~mm}$, respectively.

For this reason, we agree with previous authors that the best way to attempt to predict and prevent GTM is by adjusting the length of the tibial tunnel after careful intraoperative measurement of the length of the patella tendon. Numerous methods are described for the adjustment of the tibial tunnel, including the " $N+7$ " formula, where the length of the patella tendon in millimeters plus $7^{\circ}$ is the suggested angle of the tibial guide. ${ }^{32}$ However, this method was described for transtibial techniques, and has not been shown to eliminate GTM. ${ }^{3,4}$ Although increasing the tibial angle will tend to increase the tibial tunnel length, ${ }^{4}$ we believe it may not be completely reliable because of variations in hand position ${ }^{33}$-if a surgeon drops their hand, the tunnel becomes longer, and if they raise their hand, the tunnel becomes shorter.

Olszewski et al. described a high degree of success using a $\mathrm{N}+2 \mathrm{~mm}$ method for transtibial technique, whereby the ideal tibial tunnel length was equal to the patella tendon length plus $2 \mathrm{~mm}^{3}$. We agree with this concept and believe that using the tibial jig to more accurately estimate the tunnel length prior to drilling is important-surgeons should aim to drill a tibial tunnel length that is within $5 \mathrm{~mm}$ of the length of the patella tendon $(\mathrm{N}-5 \mathrm{~mm})$, when taking a $30-\mathrm{mm}$ tibial bone block that prevents grafts that are too short, and accounts for the IAD. In this way, small discrepancies between tunnel length and graft length that remain after application of these techniques can be addressed with femoral tunnel recession, thus avoiding the need for rescue techniques, such as trough fixation or free bone plugs.

The main variable in anatomic ACLR using BPTB other than tendon length is the IAD, with previous 
literature reporting means of $26.3 \mathrm{~mm}$ (range: 21-33 $\mathrm{mm})^{6}$ and $20.44 \mathrm{~mm}$ (range: 16-26 mm). ${ }^{31}$ In our study, the mean IAD at $30^{\circ}$ was $23.4 \mathrm{~mm}$ (range: 18-31 $\mathrm{mm})$, which is likely affected by differing femoral tunnel positions when using anteromedial portal drilling in comparison to transtibial. ${ }^{11}$ It is also important to note that the IAD is significantly shorter than the native ACL ligament (range: $27-38 \mathrm{~mm}$ ), but it was consistent with the described length of the posterolateral bundle (22-30 $\mathrm{mm}),{ }^{34}$ which is typically reconstructed when performing ACLR using anteromedial portal drilling. We also found that the IAD (and the total tunnel distance) varied with knee position, becoming longer as the knee extends, which is likely because of graft anisometry encountered in anatomic ACLR. ${ }^{13}$ While the combination of a long patella tendon and a short IAD has been described as a risk for GTM, ${ }^{35}$ we believe that the IAD is much less variable-in our study, $85 \%$ were between 18 and $25 \mathrm{~mm}$. Although techniques exist that enable the IAD to be measured without drilling the tibial tunnel first, allowing the tibial tunnel to be drilled in accordance with the graft length, ${ }^{6,31}$ unless the tibial tunnel is sufficiently long, there will be instances in which femoral tunnel drilling will not be sufficient to eliminate GTM.

\section{Limitations}

Limitations include the lack of clinical outcome scores for patients in this study, as this study was primarily a technique paper. We did not measure reliability of measurements, as this was done in a previous study. ${ }^{17}$ Although the results of this study demonstrate acceptable reliability, it is likely that there is some variation, which can possibly contribute to surgeons encountering mismatch. There were a relatively small number of patients who had GTM in this series, limiting analysis. We did not measure the patella tendon length on magnetic resonance imaging, as it was felt that these

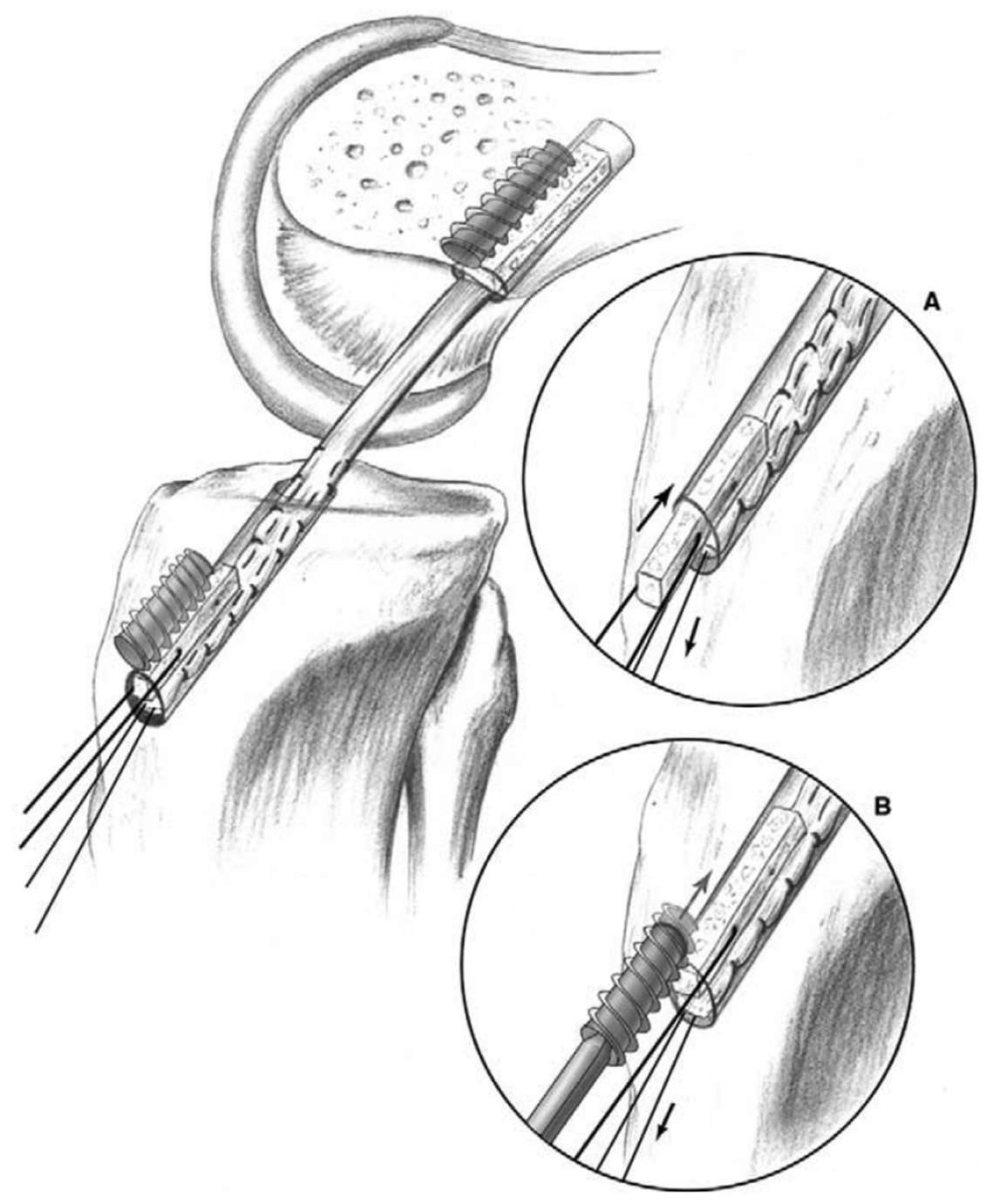

Fig 4. Management of graft-tunnel mismatch when the majority of the tibial bone block is protruding with free bone block technique (Reproduced with permission). 
measurements were done more accurately intraoperatively, without compromising surgical technique. For the same reason, patient weight and height were not recorded. Furthermore, we were not able to measure the IAD at $0^{\circ}$, as this is not technically feasible. In this study, a $30-\mathrm{mm}$ tibial bone block was taken to prevent GTM with a short graft-it is important that surgeons carefully note the length of the tibial bone block before measuring and determining any degree of GTM. It is also not possible to control for all variations in technique between all cases, and between two surgeons, despite similarity in technique. Furthermore, the techniques to avoid mismatch described in this article may not be generalizable to other ACL reconstruction techniques using BРTB.

\section{Conclusion}

The incidence of graft-tunnel mismatch after anatomic ACLR using BTPB and anteromedial portal drilling in this study is $6 \%$. To limit the occurrence of GTM for cases in which the graft is too long, surgeons should drill tibial tunnel distances within $5 \mathrm{~mm}$ of the patella tendon length.

\section{References}

1. Goldstein JL, Verma N, McNickle AG, et al. Avoiding mismatch in allograft anterior cruciate ligament reconstruction: Correlation between patient height and patellar tendon length. Arthroscopy 2010;26:643-650.

2. Verma NN, Dennis MG, Carreira DS, et al. Preliminary clinical results of two techniques for addressing graft tunnel mismatch in endoscopic anterior cruciate ligament reconstruction. J Knee Surg 2005;18:183-191.

3. Olszewski AD, Miller MD, Ritchie JR. Ideal tibial tunnel length for endoscopic anterior cruciate ligament reconstruction. Arthroscopy 1998;14:9-14.

4. Pagnano MW, Kim CW, Huie G, et al. Difficulties with the "N + 7 rule" in endoscopic anterior cruciate ligament reconstruction. Arthroscopy 1997;13:597-599.

5. Brown JA, Brophy RH, Franco J, et al. Avoiding allograft length mismatch during anterior cruciate ligament reconstruction: Patient height as an indicator of appropriate graft length. Am J Sports Med 2007;35:986-989.

6. Shaffer B, Gow W, Tibone JE. Graft-tunnel mismatch in endoscopic anterior cruciate ligament reconstruction: A new technique of intraarticular measurement and modified graft harvesting. Arthroscopy 1993;9:633-646.

7. Golish SR, Baumfeld JA, Schoderbek RJ, et al. The effect of femoral tunnel starting position on tunnel length in anterior cruciate ligament reconstruction: A cadaveric study. Arthroscopy 2007;23:1187-1192.

8. Bedi A, Raphael B, Maderazo A, et al. Transtibial versus anteromedial portal drilling for anterior cruciate ligament reconstruction: A cadaveric study of femoral tunnel length and obliquity. Arthroscopy 2010;26:342-350.

9. Lee SS, Seo IW, Cho MS, et al. Comparison of femoral tunnel length and obliquity of anatomic versus nonanatomic anterior cruciate ligament reconstruction: A meta-analysis. PLoS One 2020;15:e0230497.

10. Osti M, Krawinkel A, Ostermann M, et al. Femoral and tibial graft tunnel parameters after transtibial, anteromedial portal, and outside-in single-bundle anterior cruciate ligament reconstruction. Am J Sports Med 2015;43:2250-2258.

11. Venosa M, Delcogliano M, Padua R, et al. Femoral tunnel positioning in anterior cruciate ligament reconstruction: Anteromedial portal versus transtibial technique-A randomized clinical trial. Joints 2017;5:34-38.

12. Lee JS, Kim TH, Kang SY, et al. How isometric are the anatomic femoral tunnel and the anterior tibial tunnel for anterior cruciate ligament reconstruction? Arthroscopy 2012;28:1504-1512:1512 e1501-1502.

13. Kim YK, Yoo JD, Kim SW, et al. Intraoperative graft isometry in anatomic single-bundle anterior cruciate ligament reconstruction. Knee Surg Relat Res 2018;30:115-120.

14. Boddu CK, Arif SK, Hussain MM, et al. Prevention of graft-tunnel mismatch during anatomical anterior cruciate ligament reconstruction using a bone-patellar tendonbone graft. Bone Joint J 2015;97-B:324-328.

15. Saltzman BM, Varkey DT, Trofa DP, et al. An update on graft-tunnel mismatch in anterior cruciate ligament reconstruction: A survey of the experts in the field of orthopedic sports medicine demonstrates no clear consensus in management. Knee 2020;27:1525-1533.

16. MacDonald P, Kim C, McRae S, et al. No clinical differences between anteromedial portal and transtibial technique for femoral tunnel positioning in anterior cruciate ligament reconstruction: A prospective randomized, controlled trial. Knee Surg Sports Traumatol Arthrosc 2018;26:1335-1342.

17. Dwyer T, Bristow L, Bayley N, et al. Graft-tunnel mismatch in endoscopic ACL reconstruction: Reliability of measuring tunnel lengths and intra-articular distance. Orthop J Sports Med 2018;6:2325967118816317.

18. Pomeroy G, Baltz M, Pierz K, et al. The effects of bone plug length and screw diameter on the holding strength of bone-tendon-bone grafts. Arthroscopy 1998;14:148-152.

19. Fowler BL, DiStefano VJ. Tibial tunnel bone grafting: a new technique for dealing with graft-tunnel mismatch in endoscopic anterior cruciate ligament reconstruction. Arthroscopy 1998; 14:224-228.

20. Taylor DE, Dervin GF, Keene GC. Femoral bone plug recession in endoscopic anterior cruciate ligament reconstruction. Arthroscopy 1996;12:513-515.

21. Auge WK 2nd, Yifan K. A technique for resolution of graft-tunnel length mismatch in central third bonepatellar tendon-bone anterior cruciate ligament reconstruction. Arthroscopy 1999;15:877-881.

22. Novak PJ, Wexler GM, Williams JS Jr, et al. Comparison of screw post fixation and free bone block interference fixation for anterior cruciate ligament soft tissue grafts: biomechanical considerations. Arthroscopy 1996;12:470-473.

23. Gaines EB, Lau D, Naziri Q, et al. A biomechanical analysis of tibial ACL reconstruction with graft length mismatch. J Orthop Surg (Hong Kong) 2017;25: 2309499017690983.

24. Gerich TG, Cassim A, Lattermann C, et al. Pullout strength of tibial graft fixation in anterior cruciate ligament replacement with a patellar tendon graft: Interference 
screw versus staple fixation in human knees. Knee Surg Sports Traumatol Arthrosc 1997;5:84-88.

25. Grawe B, Smerina A, Allen A. Avoiding graft-tunnel length mismatch in anterior cruciate ligament reconstruction: the single-bone plug technique. Arthrosc Tech 2014;3:417-420.

26. Barber FA. Flipped patellar tendon autograft anterior cruciate ligament reconstruction. Arthroscopy 2000;16:483-490.

27. Matthews LS, Lawrence SJ, Yahiro MA, et al. Fixation strengths of patellar tendon-bone grafts. Arthroscopy 1993;9:76-81.

28. Hartman GP, Sisto DJ. Avoiding graft-tunnel mismatch in endoscopic anterior cruciate ligament reconstruction: a new technique. Arthroscopy 1999;15:338-340.

29. Morgan CD, Kalmam VR, Grawl DM. Isometry testing for anterior cruciate ligament reconstruction revisited. Arthroscopy 1995;1 1:647-659.

30. Kenna B, Simon TM, Jackson DW, et al. Endoscopic ACL reconstruction: a technical note on tunnel length for interference fixation. Arthroscopy 1993;9:228-230.
31. Denti M, Bigoni M, Randelli P, et al. Graft-tunnel mismatch in endoscopic anterior cruciate ligament reconstruction. Intraoperative and cadaver measurement of the intra-articular graft length and the length of the patellar tendon. Knee Surg Sports Traumatol Arthrosc 1998;6:165-168.

32. Miller MD, Hinkin DT. The "N +7 rule" for tibial tunnel placement in endoscopic anterior cruciate ligament reconstruction. Arthroscopy 1996;12:124-126.

33. Wallace M, Bedi A, Lesniak BP, et al. What effect does anterior cruciate ligament tibial guide orientation have on tibial tunnel length? Arthroscopy 2011;27:803-808.

34. Cone SG, Howe D, Fisher MB. Size and shape of the human anterior cruciate ligament and the impact of sex and skeletal growth: A systematic review. JBJS Rev 2019;7:e8

35. Mariani PP, Calvisi V, Margheritini F. A modified bonetendon-bone harvesting technique for avoiding tibial tunnel-graft mismatch in anterior cruciate ligament reconstruction. Arthroscopy 2003;19:E3. 